\title{
Farmaci innovativi e sostenibilità
}

\author{
Paolo Bonarettiti,2 \\ ${ }^{1}$ Consigliere per le politiche industriali del Ministero dello Sviluppo Economico, Roma - Italy \\ ${ }^{2}$ Direttore Generale ASTER S. Cons. p. A., Bologna - Italy
}

\begin{abstract}
Innovative drugs and sustainability
The recent availability of innovative, costly cancer drugs has prompted the search for a sustainable health economics model that would allow universal access to treatment coupled with long-term viability of the national health system.

The huge R\&D investment and the relatively short life of drugs, which must face not only the market entry of competitor products but also a growing trend to limit the type and number of patients in whom their use is allowed, are the main factors underlying the high unit cost of innovative drugs; this ultimately has a major impact on overall drug expenditure, especially for in-hospital treatment.

To facilitate the inclusion of innovative drugs and maximize their beneficial effect on patients, we need to increase the efficiency of the national health system overall, and be able to measure the costs that can be avoided through a coherent HTA system apt to leverage synergies at a regional level, thereby freeing wasted resources that can be better invested in this area.

Secondly, the continuous monitoring of an innovative drug throughout its life cycle, based on the correct comparative evaluation of its efficacy for each therapeutic indication, can lead to a more refined definition of drug prices. Furthermore, cost containment must in all instances include appropriate negotiation of the unit price based on the overall volume acquired.

Last but not least, a significant R\&D investment in clinical research is a fundamental step that can leverage synergies between public funds and contributions from pharma companies, allowing to overcome systems based on ceilings and/or payback models that, in the current scenario, appear undoubtedly outdated.
\end{abstract}

Keywords: Big data, Clinical data, Clinical and pharmacological research, Drug expenditure, Health expenditure, Innovative drugs, Investment, Sustainability

La spesa farmaceutica aumenta in tutto il mondo (1). I fattori sono molteplici: lo sviluppo demografico e l'urbanizzazione nei Paesi di recente sviluppo, l'invecchiamento della popolazione in Europa e lo sviluppo di nuovi farmaci innovativi ad alto costo. In Italia la spesa farmaceutica complessiva ha raggiunto i 25 miliardi di euro, di cui 18 nell'ambito del SSN (Sistema Sanitario Nazionale), mentre la rimanente parte rientra nei 35 miliardi di spesa sanitaria cosiddetta out of pocket, cioè a carico delle famiglie (2). Tale dimensione ha determinato uno sforamento di circa 1,8 miliardi del tetto complessivo della spesa farmaceutica rimborsata dal SSN,

Accepted: July 12, 2016

Published online: September 27, 2016

Indirizzo per la corrispondenza:

Paolo Bonaretti

Direttore Generale ASTER S. Cons. p. A.

Via Gobetti 101

40129 Bologna, Italy

paolo.bonaretti@aster.it per la maggior parte imputabile alla spesa ospedaliera che ha raggiunto i 5,5 miliardi (oltre il 5\% del Fondo SSN a fronte di un tetto previsto del 3,5\%). Quindi negli ultimi anni la spesa farmaceutica pubblica è aumentata al ritmo di 500 milioni di euro/anno. Per i prossimi anni, seppure a ritmi inferiori è comunque previsto un ulteriore incremento.

L'aumento è dovuto in gran parte all'ingresso di farmaci innovativi ad alto costo unitario imputabile a tre essenziali ordini di fattori:

1. I costi della ricerca: una nuova molecola sul mercato ha un costo di circa 2 miliardi, compresi i costi dei fallimenti e dei mancati investimenti finanziari alternativi. Tale investimento viene solitamente finanziato con l'accesso al mercato finanziario del capitale di rischio, che di norma richiede tempi di rientro inferiori agli oltre 10 anni del time to market dello sviluppo di un prodotto farmaceutico, e rendimenti superiori al mercato del credito.

2. L'accorciarsi del tempo del ciclo di vita del prodotto, non più legato principalmente alla durata brevettuale, ma sempre più spesso determinato dal prodotto concorrente più performante che arriva sul mercato in tempi rapidissimi, mediamente 2-3 anni. 
3. La sempre maggiore focalizzazione dei farmaci, specie biologici, che limitano inizialmente la numerosità del target dei pazienti per indicazione terapeutica, salvo poi moltiplicare le indicazioni stesse.

4. Alle modalità di raccolta dei finanziamenti per la ricerca farmaceutica, attraverso i mercati finanziari che stimolano comportamenti speculativi. Semplificando, le molecole diventano degli assetti patrimoniali delle aziende determinandone significativamente il valore delle azioni.

Bisogna infatti considerare che, per esempio, le immunoterapie, che costituiscono parte rilevante dei nuovi farmaci innovativi, non aggrediscono direttamente la patologia, ma stimolano la reazione dei sistemi immunitari; altri intervengono su biologie cellulari e su modifiche del DNA, con tecnologie spesso, in parte o in toto replicabili.

Pertanto quasi sempre, dopo una prima registrazione, vengono successivamente registrati per diverse (a volte molte) indicazioni terapeutiche.

L'innovatività di questi farmaci, il loro costo, la flessibilità di utilizzo e soprattutto la velocità con cui si susseguono le innovazioni, rendono necessario un nuovo paradigma di approccio al farmaco da parte del SSN (come da parte anche di tutti i sistemi assicurativi o mutualistici, anche privati).

Innanzitutto il prezzo del farmaco deve essere valutato sulla base dell'efficacia, degli outcome e nella definizione del costo per il SSN, e dunque il rimborso deve essere valutato nell'insieme della "cura": ospedalizzazione e non, device, assistenza ecc.

Devono essere valutati i costi evitati ed evitabili, attraverso un sistema unitario di HTA (Health Technology Assessment) e di valutazioni di impatto a carattere nazionale che valorizzi competenze e attività delle Regioni. A tal fine non è possibile prescindere dal superamento della logica dei budget per silos, a partire quantomeno dalle spese di investimento di tutto il sistema sociosanitario.

Fondamentale sarà valutare diversamente l'innovatività del farmaco.

Deve infatti essere considerata la reale innovatività sulla base di miglioramenti molto significativi (QALY - Quality-Adjusted Life Year, outcome...).

L'innovatività deve essere altresì valutata comparativamente ogni qualvolta arriva sul mercato un farmaco nuovo con la medesima indicazione e quindi deve essere, nel caso, creato un percorso di uscita del farmaco dall'ambito dell'innovatività, ridefinendo e rinegoziando il prezzo (3).

Di fatto si potrebbe presumere che all'arrivo di un nuovo farmaco più performante, vi sia l'uscita di un vecchio farmaco dalla lista degli innovativi. Considerando però la pluralità di indicazioni, potrebbe esserci un effetto di sostituzione verticale solo parziale, accompagnato da un effetto di sommatoria orizzontale dei farmaci innovativi. Tale considerazione spinge sempre più verso la definizione del costo per "cura" (una nuova tipologia di DRG - DiagnosisRelated Group), non per farmaco.

Soprattutto sarà necessaria, comunque e in ogni caso, una forte correlazione inversa tra costo unitario e volume fisico e/o di fatturato, una contrattazione prezzo-volume generalizzata. II criterio prezzo-volume dovrebbe divenire il principale strumento di contenimento dei costi, sostituendo gradualmente, in combinazione con l'inserimento del farmaco nel DRG, l'attuale sistema dei tetti per la spesa farmaceutica ospedaliera.

Due considerazioni debbono essere fatte a questo punto: sul sistema dei "registri" e sul fondo per i farmaci innovativi (4).

Per realizzare un sistema come quello sopra descritto, specie per i farmaci ad alta innovatività, $m a$ in generale per tutti i nuovi farmaci, dovrà essere implementato e rafforzato anche qualitativamente il sistema dei registri farmaceutici AIFA (Agenzia Italiana del Farmaco). I registri dovranno corrispondere all'esigenza di una valutazione continua (o in itinere) della efficacia e degli outcome del farmaco, consentendone anche una valutazione comparativa per indicazione terapeutica, con i farmaci in ingresso sul mercato, cioè una valutazione in continuo dell'innovatività, ai fini della permanenza nella lista degli innovativi e della conseguente ridefinizione del prezzo. Va da sé poi, che il sistema dei registri debba operare in continuità con i dossier registrativi, misurando le variazioni di efficacia e outcome, rispetto ai target definiti nella negoziazione iniziale.

Ovviamente il sistema dei registri, come già avviene per alcuni farmaci innovativi, dovrà garantire un flusso costante di dati certificati sui volumi di vendita, e su ogni aspetto amministrativo e di tracciabilità. A tale proposito pare opportuno che i flussi dei dati farmaceutici vengano convogliati direttamente ad AIFA, il soggetto che deve gestirli, superando l'attuale incertezza sulla qualità dei dati.

II Fondo per gli innovativi deve essere rafforzato e reso permanente anche, e forse soprattutto, in una prospettiva di inserimento del valore dei farmaci nel DRG, o comunque in una forma di valorizzazione di costo della cura. Infatti un nuovo farmaco non può essere inserito nel DRG prima di una valutazione in real life di almeno 2-3 anni dopo la registrazione e l'ingresso sul mercato. II Fondo Innovativi deve dunque diventare un buffer, nel quale costantemente entrano nuovi farmaci veramente innovativi e dal quale, con eguale continuità, escono i farmaci che innovativi non sono più. Al tempo stesso deve divenire il luogo di elezione della valutazione della innovatività e di sviluppo dei nuovi metodi di rimborso condizionato e della loro evoluzione.

In tale prospettiva è evidente la centralità di AIFA, che deve essere rafforzata sul piano quali-quantitativo, come peraltro già previsto dalle norme attuali. Un passo ulteriore deve però essere fatto: i risultati molto positivi di AIFA, in comparazione con gli altri casi europei e internazionali, sono in gran parte dovuti al fatto di avere concentrato nel medesimo soggetto regolazione, valutazione scientifica e responsabilità negoziale. Unificare comitato scientifico e comitato prezzi, organizzandone il lavoro in modo continuo e permanente, consentirebbe da un lato di accorciare sensibilmente i tempi delle approvazioni e delle decisioni, e dall'altro di avere un organismo dedicato a tempo pieno a garantire quella continuità delle valutazioni scientifiche e di impatto, che sta alla base di un nuovo sistema di governance della spesa, flessibile e sostenibile. 


\section{Attrarre nuove risorse}

Anche in presenza di una politica aggressiva di contenimento della spesa, difficilmente si potrà pensare a un sistema sostenibile affrontandolo dal solo lato dei costi: dobbiamo valorizzare il sistema della salute nazionale come un attrattore di investimenti e risorse, in termini economici e finanziari, secondo quattro filoni principali:

1. la realizzazione di un HTA che definisca meccanismi precisi (innanzitutto in ambito sanitario, assistenziale e previdenziale), una compensazione in termini economici dei costi evitati;

2. un meccanismo di "mutualità tra sistemi" che, in particolare negli eventi a maggior costo (no autosufficienza), determini forme di sostegno e compensazione reciproca tra SSN e Fondi/Assicurazioni;

3. l'attrazione di investimenti in ricerca, e in particolare in ricerca clinica. II mercato della ricerca farmaceutica vale nel mondo circa 160 miliardi di dollari/anno. L'ltalia ha un buon posizionamento nella ricerca di base e la ricerca clinica viene giudicata di ottima qualità. II collo di bottiglia sta nel sistema burocratico, autorizzatorio (comitati etici, contrattualistica, tempi, formazione degli operatori, controlli di qualità).

Le imprese, multinazionali incluse, convergono sul fatto che rimossi tali ostacoli, si potrebbero duplicare o triplicare gli investimenti in ricerca clinica (cioè almeno 2 miliardi di investimento aggiuntivo sulla ricerca e sul Sistema Sanitario Nazionale). Si aggiunga poi che nelle fasi della ricerca clinica i pazienti vengono curati gratuitamente e i Centri che svolgono ricerca clinica sono quelli avvantaggiati nell'accesso per "uso compassionevole" (sempre gratuito) del farmaco nel periodo che precede immediatamente la fase commerciale. Per ottenere questo risultato è necessario:

- Avviare rapidamente il cosiddetto Fast Track elaborato presso il Ministero della Salute (un agreement a base volontaria sull'utilizzo di un unico Comitato etico negli studi multicentrici, tempi certi per le diverse fasi, condivisione delle conoscenze e della formazione, contrattualistica comune). Parallelamente, con una norma, realizzare una rete di pochi (massimo 10-15) Comitati etici dedicati unicamente alla ricerca clinica, specializzati per aree terapeutiche.

- Investire sulle infrastrutture comuni e le reti per la ricerca, la produzione innovativa, lo sviluppo di startup biotecnologiche, rafforzando settori di maggiori competenze e più promettenti (terapie geniche e cellulari, nuovi vaccini, immuno-terapici e immuno-oncologici) utilizzando per esempio gli strumenti del Piano Junker o dell'Istituto Nazionale di Promozione.

- Sviluppare un sistema di registri farmaceutici di altissima qualità (oggi è comunque buono) che sia comune alla fase di registrazione del farmaco e alla fase degli studi osservazionali in real life negli anni successivi, al fine di verificare l'efficacia e le relative condizioni di variabilità dell'efficienza, e quindi consentire studi comparativi, e dunque la rinegoziazione continua del costo rimborsabile. Un sistema di registri con queste caratteristiche potrebbe avere un valore mondiale.

- Sviluppare una forma di PPP (Public Private Partnership) sulla ricerca clinica, sotto il controllo o con la golden share di ISS (Istituto Superiore di Sanità) come autorità scientifica e AIFA come autorità regolatoria e di controllo, con la partecipazione dell'industria, delle organizzazioni no profit, del sistema della ricerca clinica nazionale a partire dalle reti degli IRCCS (Istituti di Ricovero e Cura a Carattere Scientifico). Con l'obiettivo di creare un ecosistema condiviso per potenziare e far convergere la ricerca clinica indipendente, fare lo scouting e promuovere la valorizzazione della ricerca biomedica e biotecnologica italiana, studiare i miglioramenti possibili nei sistemi organizzativi, della qualità dei dati e dei registri, sviluppare attività formative congiunte e standardizzate, sviluppare modelli contrattuali e assicurativi condivisi, promuovere e progettare infrastrutture di ricerca e sviluppo clinico ecc.

Alla PPP le industrie dovrebbero contribuire in forma "incondizionata" pur partecipando agli indirizzi generali e agli specifici gruppi di lavoro.

4. Valorizzazione del dato sanitario, big data e attrazione di risorse per il SSN.

II SSN genera e dispone di una enorme quantità di dati con una granularità finissima: anagrafici e amministrativi, i registri farmaceutici, terapeutici, per patologia, sulla storia clinica, sugli eventi ospedalieri, sul consumo dei farmaci, sulle condizioni sociali. Molti di questi dati sono destrutturati.

Sono una quantità di set di dati di qualità in capo a un soggetto unitario, probabilmente unica al mondo, proprio dovuta alla caratteristica universale del nostro SSN, che comprende attraverso l'accreditamento, anche la maggior parte del sistema privato.

La strutturazione di questi dati, ma anche l'uso in forma destrutturata (pur rispettando rigorosamente la privacy) ha potenzialmente un valore economico enorme (anche qui nell'ordine di miliardi) sia per gli studi di tipo epidemiologico e clinico sull'applicabilità e l'efficienza delle cure e dei farmaci, sia per ottimizzare e sviluppare investimenti sulla gestione amministrativa e finanziaria, ma soprattutto a fini predittivi.

Le nuove tecnologie di calcolo computazionale cognitivo consentono infatti di utilizzare i dati, anche destrutturati, per sviluppare algoritmi di sistemi predittivi dell'efficacia, sugli impatti dello sviluppo epidemiologico, delle capacità e delle traiettorie delle relative mutazioni, ma anche dei comportamenti degli stili di vita, alimentari ecc. In grado quindi di valutare anche gli impatti impliciti delle innovazioni (5).

Del resto anche le ipotesi di screening delle popolazioni a rischio attraverso il sequenziamento massivo del genoma (oggi possibili per la riduzione in scala logoritmica dei costi) contribuiscono ad accrescere que- 
sta potenzialità. Lo sviluppo di Human Technopole e il recente protocollo siglato con IBM vanno in questa direzione (6).

È dunque necessario immediatamente strutturare una iniziativa per la valorizzazione del "dato sanitario" che veda assieme al SSN, il Governo e le Regioni, in una collaborazione stretta con le associazioni dei pazienti, e sul piano tecnologico con il sistema delle imprese e della ricerca a partire da Human Technopole, che va inteso come polo di sviluppo di ricerca di eccellenza di un sistema a rete della ricerca nazionale a partire dagli IRCCS, e al tempo stesso fattore di attrattività di imprese innovative e talenti.

In conclusione, la riflessione sullo sviluppo e la sostenibilità dei farmaci innovativi impone un salto di paradigma, nell'organizzazione della governance farmaceutica, negli approcci alle cure, nell'organizzazione della ricerca. II salto forse più difficile, ma indispensabile e indifferibile, sta nel considerare il SSN non più unicamente come un centro di spesa erogatore di assistenza, ma anche come attrattore di risorse e investimenti, motore di crescita, innovazione, ricerca e lavoro qualificato.

\section{Disclosures}

Financial support: No financial support was received for this submission. Conflict of interest: The author has no conflict of interest with this submission.

\section{Bibliografia}

1. OECD, Health at a Glance 2015: OECD Indicators, Paris: OECD Publishing, 2015. http://dx.doi.org/10.1787/health_glance-2015-en.

2. Osservatorio Nazionale sull'impiego dei Medicinali. L'uso dei farmaci in Italia. Rapporto Nazionale gennaio-settembre 2015. Roma: Agenzia Italiana del Farmaco, 2016.

3. Fonte AIFA, Agenzia Italiana del Farmaco. Criteri di valutazione per l'attribuzione del grado di innovazione terapeutica http:// www.agenziafarmaco.gov.it/it/content/criteri-di-valutazione. Ultimo accesso: 26 aprile 2016.

4. AlFA, Agenzia Italiana del Farmaco. Lista aggiornata dei Registri e dei Piani Terapeutici web-based http://www.agenziafarmaco.gov.it/it/content/lista-aggiornata-dei-registri-e-dei-pianiterapeutici-web-based.

5. Disponibile sul sito: http://www.iss.it/binary/publ/cont/14_ 23_pt_1_web.pdf.

6. Disponibile sul sito: http://www.governo.it/articolo/dopo-expo-presentato-il-progetto-di-ricerca-human-technopole-italy2040/4205. 